\title{
Progress of the Volume FEL (VFEL) experiments in millimeter range ??
}

\author{
V.G.Baryshevsky K.G.Batrakov, A.A.Gurinovich, I.I.Ilienko, \\ A.S.Lobko, P.V.Molchanov, V.I.Moroz, P.F.Sofronov, \\ V.I.Stolyarsky. \\ Research Institute of Nuclear Problems, Belarussian State University, \\ 11 Bobruyskaya Str., Minsk 220050, Belarus
}

\begin{abstract}
Use of non one-dimensional distributed feedback in VFEL gives possibility of frequency tuning in wide range. In present work dependence of lasing process on the angle between resonant diffraction grating grooves and direction of electron beam velocity is discussed.
\end{abstract}

Key words: Volume Free Electron Laser (VFEL), Volume Distributed Feedback (VDFB), diffraction grating, Smith-Purcell radiation, electron beam instability PACS: 41.60.C, 41.75.F, H, 42.79.D

\section{Introduction}

New advances in different areas require the development of tunable, wideband, high-power sources of coherent electromagnetic radiation in GigaHertz, TeraHertz and higher frequency ranges. Conventional electron vacuum devices have restricted possibility of frequency tuning (usually it does not exceed 5$10 \%$ ) for the certain carrier frequency at certain e-beam energy. Volume free electron laser (VFEL) [3,4] was proposed as a new type of free electron laser. Frequency tuning, possibility of use of wide electron beams (several e-beams) and reduction of threshold current density necessary for start of generation,

\footnotetext{
* This work is carried out with financial support of private joint-stock company BelTechExport, Belarus
} 
provided by VFEL, make it a basis for development of more compact, highpower and tunable radiation sources then conventional electron vacuum devices could let.

First lasing of VFEL was reported at FEL 2001 [4].

\section{Volume FEL distinctive features}

Benefits given by VFEL:

1. Volume FEL provides frequency tuning by rotation of diffraction grating;

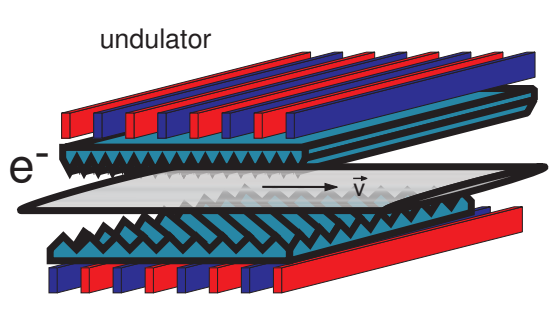
one-dimensional distributed feedback

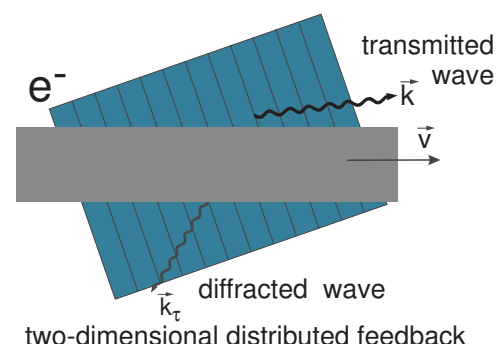

two-dimensional distributed feedback

Fig. 1.

2. Use of multi-wave diffraction reduces generation threshold and the size of generation zone. Starting current $\mathrm{j}$ depends on interaction length $\mathrm{L}$ as [2]: $j_{\text {start }} \sim 1 /\left\{(k L)^{3}\left(k \chi_{\tau} L\right)^{2 s}\right\}, s$ is the number of surplus waves appearing due to diffraction (for example, in case of two-wave Bragg diffraction $s=1$, for three-wave diffraction $s=2$ and so on).

\section{3-wave diffraction ( $s=2)$}

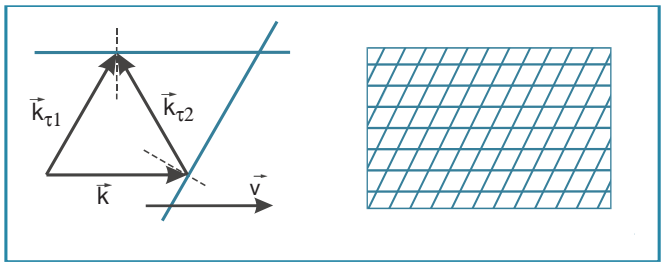

6-wave diffraction (s=5)

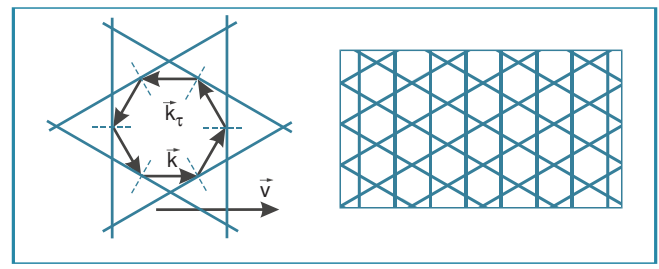

Fig. 2.

3. Wide electron beams and diffraction gratings of large volumes can be used in VFEL. Two or three-dimensional diffraction gratings allow to distribute interaction over large volume and to overcome power restrictions in resonator. Volume distributed feedback provides mode discrimination in VFEL resonator. 

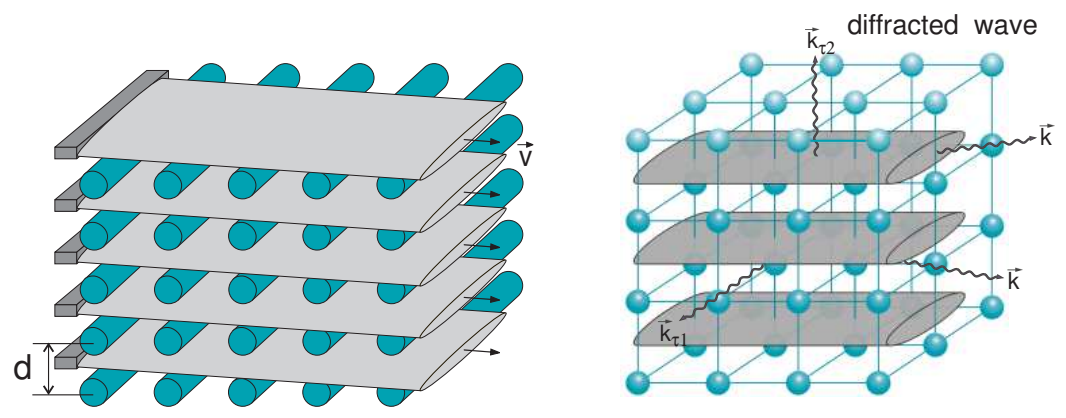

Fig. 3.

4. VFEL can simultaneously generate radiation at several frequencies that is extremely important, for example, for development of multifrequency radiation source.

\section{Experimental setup}

VFEL operates in wavelength range $\sim 4 \mathrm{~mm}$ at electron beam energy up to $10 \mathrm{keV}$. Such generating device is very interesting for practical use in submillimeter (TeraHertz) range, which is actively employing for medical examination. VFEL resonator is formed by two parallel diffraction gratings with different periods and two smooth sidewalls (see figure in [4]). The interaction of the exciting diffraction grating with an electron beam arouses Smith-Purcell radiation. The resonant diffraction grating provides distributed feedback of generated radiation with electron beam by Bragg dynamical diffraction. Resonant grating can rotate to change orientation of grating grooves with respect to electron beam velocity that provides tuning of diffraction conditions. I
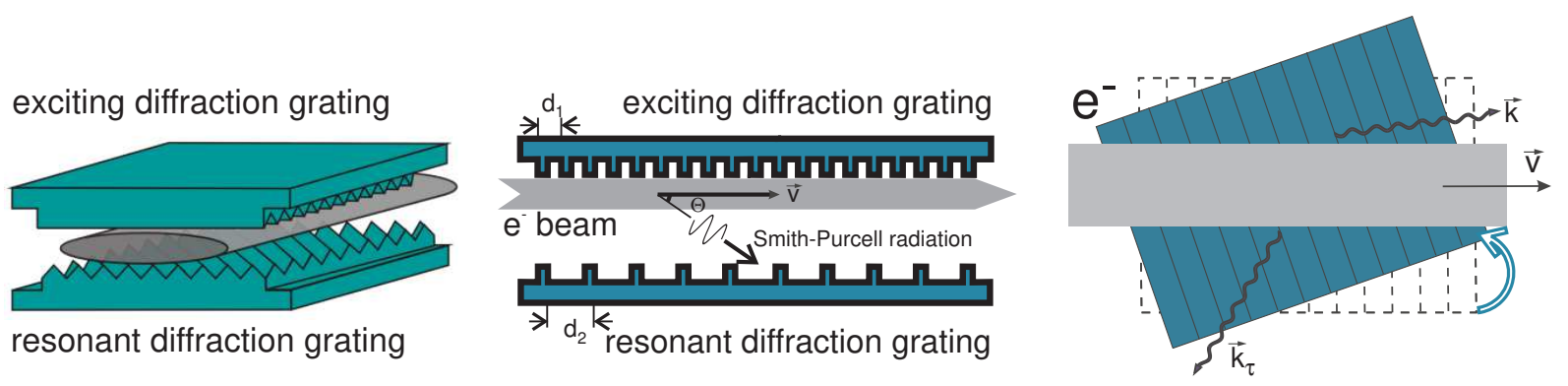

Fig. 4.

$\overline{1}$ Undoubtedly, fast frequency modulation of VFEL radiation can be done by rotation of electron beam (for example, by the use of drift in crossed fields) with respect to diffraction grating grooves. Also frequency tuning can be provided by azimuth rotation of annular electron beam in resonator formed by two cylindrical diffraction gratings with helix grooves. 
The length of resonator is $100 \mathrm{~mm}$, periods of the exciting diffraction grating and resonant diffraction grating are $0.67 \mathrm{~mm}$ and $3 \mathrm{~mm}$, respectively. The ribbon electron beam with profile $10 \mathrm{~mm} \times 1 \mathrm{~mm}$ is emitted by thermal cathode, formed in Pierce gun without beam compression and guided through resonator along dffraction gratings. Only electrons passing at the distance $h \leq \lambda \beta \gamma /(4 \pi)$ [6] near the exciting grating surface interact with electromagnetic wave effectively (i.e. less than $10 \%$ of total cross size of electron beam participates in lasing process). To provide the most effective interaction of electron beam with the exciting grating, it is placed in a beam and the value of "grating current" (i.e. part of total electron beam current falling to the grating) is monitored as a touchstone of interaction efficiency and indicator of beam position with respect to grating surface. The following parameters can be monitored during experiment: beam voltage (electron beam energy); total electron beam current (number of emitted electrons per second); "grating current"; angle between resonant grating grooves and electron beam velocity (angle of grating rotation); distance between gratings (transverse size of resonator); microwave power and frequency. Electrons are emitted in pulsed regime (unipolar pulse with sinusoidal shape and pulse duration $10 \mathrm{~ms}$ ) in sequence of two or three voltage pulses. Voltage can vary from 0 to $10 \mathrm{kV}$ (hence, electron beam energy is varied from 0 to $10 \mathrm{keV}$ ). Different longitudional modes of resonator are excited while electron energy passes through the whole variation interval. Frequency of data reading is determined by the condition that change of electron energy between two consecutive measurements of each monitored parameter should not cause change of generation regime. Estimated admissible change of energy is about $\sim 1 \%$ of rating value. Data reading interval is $4 \cdot 10^{-5} \mathrm{~s}$.

A lot of system parameters can be varied during experiment, so complete investigation of the system requires multiple measurements. Experimental study of frequency tuning and change of radiation intensity at mechanical rotation of resonant diffraction grating (i.e. at variation of the angular orientation of grooves of resonant diffraction grating with respect to electron beam velocity) is presented in this paper.

\section{Theoretical background}

General theoretical statements $[1,3]$ should be reminded for better understanding of the below experimental results. Considering processes in resonator one should discern the difference between two cases: (a) presence of resonator sidewalls can be neglected; (b) presence of sidewalls of resonator can not be neglected. For unbounded waveguide (case (a)) two geometries of diffraction are distinguished: those of Bragg and Laue [5]. Laue geometry of diffraction implies that both incident and diffracted waves passes to vacuum through the

same boundary of resonator, while at Bragg diffraction incident and diffracted 
waves passes through the different boundaries. Absolute instability can appear in Bragg geometry and such system works as generator (for backward Bragg diffraction it converts into a well-known backward-wave tube). For Laue case convective instability provides only amplification regime.

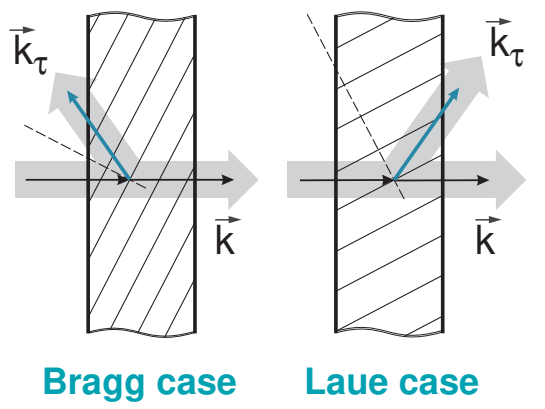

Fig. 5.

Generation process in resonator is described by Maxwell equations, containing the space-periodic permittivity of diffraction grating $\chi(\vec{r}, \omega)=\sum_{\vec{\tau} \neq 0} \chi_{\tau}(x) e^{-i \tau_{y} y} e^{-i \tau_{z} z}$, where $\vec{\tau}$ is the reciprocal lattice vector of the diffraction grating [3].

If $\chi_{\tau}$ in the above equations is equal to zero, they describe smooth waveguide, which eigenfunctions $|\vec{Y}(x, y)\rangle$ are well known. If presence of waveguide sidewalls can not be neglected, $\left(\left|\vec{Y}_{n m}(x, y)\right\rangle \sim \sin \frac{\pi n}{a} x \sin \frac{\pi m}{b} y\right.$, where $n, m$ are the integer numbers, $a$ and $b$ are the transversal dimensions of resonator).

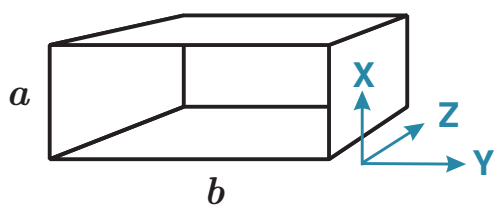

Fig. 6.

Expanding Maxwell equations over eigenfunctions of smooth waveguide $\left|\vec{Y}_{n m}(x, y)\right\rangle$ one obtains equations equations describing generation in continuous effective grating with periodic permittivity $\chi_{\text {eff }}(z)[3]$.

In single-mode approximation the above problem comes to that of generation (amplification) in one-dimensional periodic medium with effective permittivity

$$
\chi_{e f f}(z)=\chi^{n n(m m)}(z) \sim \int d x \sin ^{2} \frac{\pi n}{a} x \chi_{\tau}(x) \int d y \sin ^{2} \frac{\pi m}{b} y e^{-i \tau_{y} y} e^{-i \tau_{z} z}(1)
$$

Presence of diffraction grating in waveguide makes nondiagonal matrix elements $\chi^{n n^{\prime}\left(m m^{\prime}\right)}(z)$, describing transition between modes, different from zero:

$$
\chi^{n n^{\prime}\left(m m^{\prime}\right)}(z) \sim \int d x \sin \frac{\pi n}{a} x \chi_{\tau}(x) \sin \frac{\pi n^{\prime}}{a} x \int d y \sin \frac{\pi m}{b} y e^{-i \tau_{y} y} e^{-i \tau_{z} z} \sin \frac{\pi m^{\prime}}{b} y .
$$


Presence of sidewalls imparts new feature to diffraction process in resonator, because even in Laue-like case (which corresponds to Laue geometry of diffraction if neglecting the sidewalls) the matrix element $\chi_{\text {eff }}(z)$ differs from zero. And, as a result, generation regime can be reached at Laue-like geometry, in contrast with an unbounded waveguide, where Laue geometry provides only amplification regime, while generation is possible at Bragg diffraction.

\section{Experimental results}

General view of microwave signal is presented on Fig.7a. Presence of several microwave peaks is the manifestation of longitudinal cavity modes. Frequency difference $\Delta \nu$ associated with the change $\Delta E$ of electron energy $E$ $(\nu \sim \sqrt{E(k e V)})$ can be estimated as $\Delta \nu=\nu_{0} \frac{\Delta E}{2 E_{0}}$. According to measurements $\nu_{0} \approx 54 \mathrm{GHz}, \Delta E \approx 0,13 \mathrm{keV}$ for two next distinguished peaks (Fig.7b.), hence, frequency difference for them can be estimated as $\Delta \nu \sim 0,57 \mathrm{GHz}$.
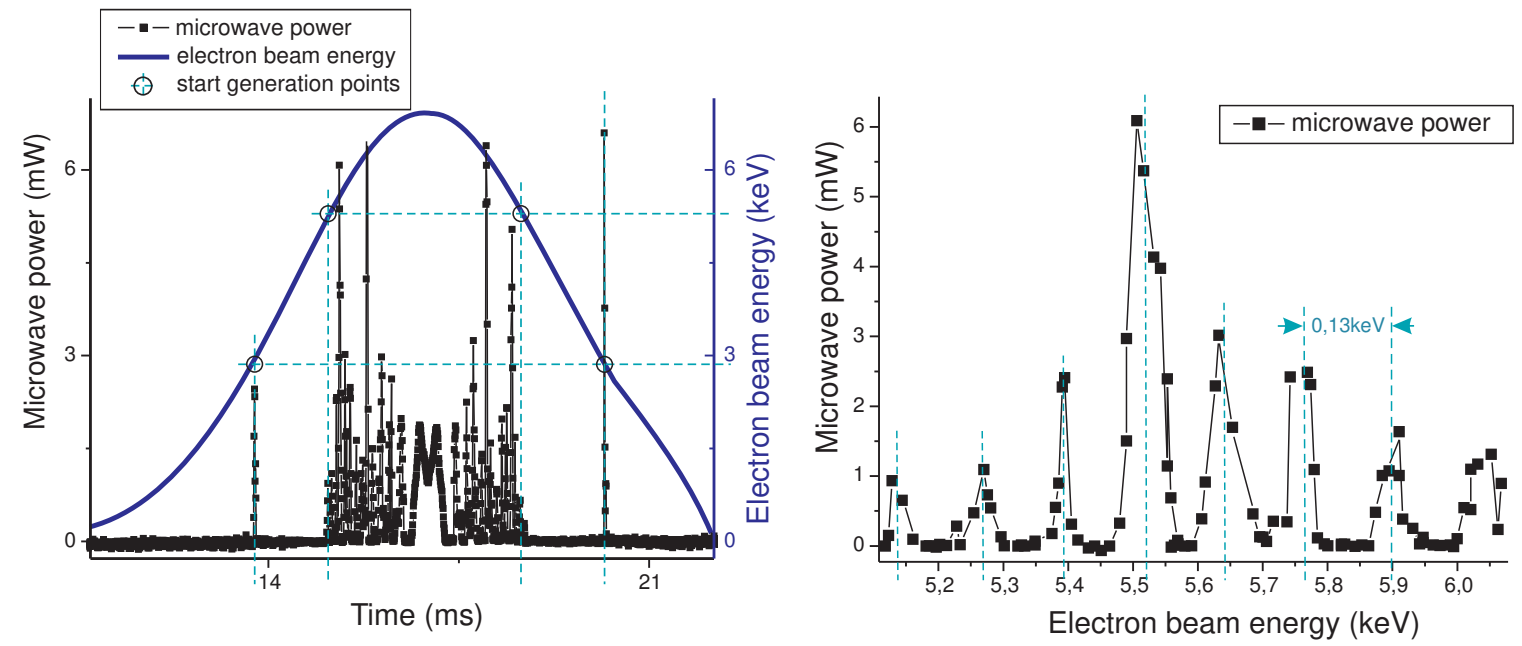

Fig. 7. a. General view of microwave signal; b. Dependence of microwave power on electron beam energy.

Now let us consider behavior of lasing efficiency with grating rotation. Rotation of diffraction grating changes components of reciprocal lattice vector. From (1) it is easy to see that $\chi_{e f f}(z)$ decreases with $\tau_{y}$ growth. Change of $\chi_{\text {eff }}(z)$ yields change of generation conditions and, in particular, generation threshold. Reduction of $\chi_{\text {eff }}(z)$ results in decrease of generation efficiency (Fig.8). But, interaction efficiency can be increased if recollect that at certain value of current density the intensity is proportional to the term $e^{-\frac{4 \pi}{\lambda \beta \gamma} h}[6]$ where $h$ is the distance from electron to the grating, $\gamma$ is the Lorentz-factor, $\lambda$ is the radiation wavelength, $\beta=v / c=\sqrt{2 E(\mathrm{keV}) / 511}$. As a result, energy 


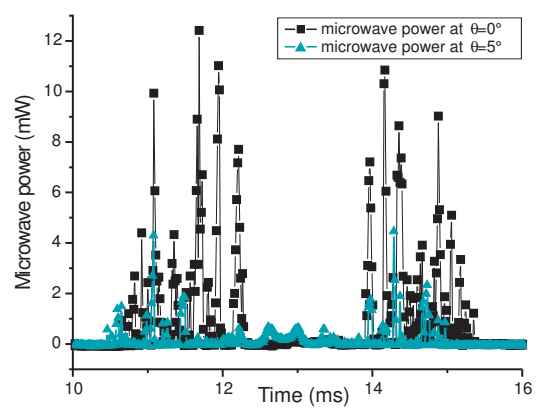

Fig. 8. Change of lasing efficiency with grating rotation, $\theta$ is the angle of grating rotation, $\theta=0$ when grating grooves are perpendicular to e-beam velocity.

of electron beam at certain current density should be increased (to increase factor $\left.e^{-\frac{4 \pi}{\lambda \beta \gamma} h}\right)$ to overcome decrease of $\chi_{e f f}$.

Non-one-dimensional feedback, being used in VFEL, provides tuning of radiation frequency by diffraction grating rotation. Measured frequencies at rotation of resonant diffraction grating for microwave peak, corresponding electron beam energy 2,9 keV are shown in Fig.9. Measured frequency change well accords theoretical predictions $[1,3]$

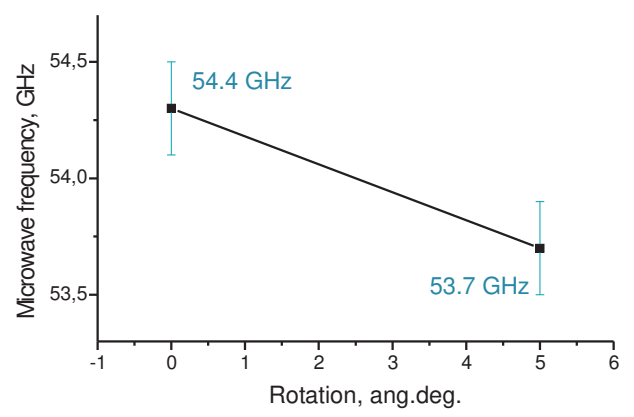

Fig. 9. Measured frequencies at rotation of resonant diffraction grating.

\section{Conclusion}

VFEL generator can be considered as a new type of tunable backward wave tube with variable period (due to gratings rotation) and VFEL amplifier is a new type of tunable travelling wave tube.

Frequency tuning, possibility of use of wide electron beams (several e-beams) and reduction of threshold current density necessary for start of generation, provided by VFEL, make it a basis for development of more compact, highpower and tunable radiation sources then conventional electron vacuum devices could let. 
New VFELs with electron beam energy up to $30 \mathrm{keV}$ and $500 \mathrm{keV}$ have been developing for next set of experiments.

\section{References}

[1] V.G.Baryshevsky, I.D.Feranchuk, Phys.Lett. 102A, 141 (1984).

[2] V.G.Baryshevsky, K.G.Batrakov, I.Ya. Dubovskaya Journ.Phys D.24 19911250.

[3] V.G.Baryshevsky, LANL e-print archive physics/9806039.

[4] V.G.Baryshevsky, K.G.Batrakov, A.A.Gurinovich, I.I.Ilienko, A.S.Lobko, V.I.Moroz, P.F.Sofronov, V.I.Stolyarsky, NIM A 483, 21 (2002).

[5] S.L. Chang, Multiple Diffraction of X-Rays in Crystals. Springer-Verlag, 1984.

[6] L.Schachter, A.Ron Phys. Rev. A 40876 (1989) 